\title{
Optical Design of a Solar Parabolic Concentrating Collector Based on Trapezoidal Reflective Petals
}

\author{
Saša Pavlović ${ }^{1}$, Velimir Stefanović ${ }^{1}$, Darko Vasiljević ${ }^{2}$ and Emina Petrović ${ }^{3}$ \\ 1. Thermal Engineering Department, Faculty of Mechanical Engineering University of Nis, Niš 18000, Serbia \\ 2. Institute of Physics, Photonics Center, Belgrade 11080, Serbia \\ 3. Mechatronic and Control System, Faculty of Mechanical Engineering University of Nis, Niš 18000, Serbia
}

Received: February 06, 2015 / Accepted: April 01, 2015 / Published: August 31, 2015.

\begin{abstract}
In this paper, detailed optical of the solar parabolic dish concentrator is presented. The system has diameter $D=2,800 \mathrm{~mm}$ and focal length $f=1,400 \mathrm{~mm}$. The efficient conversion of solar radiation in heat at these temperature levels requires a use of concentrating solar collectors. In this paper, detailed optical design of the solar parabolic dish concentrator is presented. The parabolic dish of the solar system consists from 12 curvilinear trapezoidal reflective petals. This paper presents optical simulations of the parabolic solar concentrator unit using the ray-tracing software TracePro. The total flux on receiver and the distribution of irradiance for absorbed flux on center and periphery receiver are given. The total flux at the focal region is $4,031.3 \mathrm{~W}$. The goal of this paper is to present optical design of a low-tech solar concentrator, that can be used as a potentially low-cost tool for laboratory-scale research on the medium-temperature thermal processes, cooling, industrial processes, solar cooking and polygeneration systems, etc.
\end{abstract}

Key words: Solar parabolic dish concentrator, optical analysis, solar energy, solar radiation.

\section{Introduction}

The device which is used to transform solar energy to heat is referred to a solar collector. Solar thermal collectors have been widely used to concentrate solar radiation and convert it into medium-high temperature thermal processes. They can be designed as various devices including solar cooker [1], solar hydrogen production $[2,3]$ and Dish Stirling system of harvest electricity $[4,5]$. The main types of concentrating collectors are: parabolic dish, parabolic trough, power tower, and Fresnel collector with mirror or lens and stationary concentrating collectors. The ideal optical configuration for the solar parabolic thermal concentrator is a parabolic mirror. The parabolic mirror is very expensive to fabricate and it is cost escalating rapidly with increase of aperture area. The parabolic mirror can be designed with large number of

Corresponding author: Saša Pavlović, research associate, research field: solar energy system. E-mail: saledoca@gmail.com. elementary components known as reflecting petals or facets. Usually reflecting petals are made from glass and their thickness is from $0.7 \mathrm{~mm}$ to $1.0 \mathrm{~mm}$. Traditionally, the optical analysis of radiation concentrators has been carried out by means of computer ray-trace programs. Recently, an interesting analytical solution for the optical performance of parabolic dish reflectors with flat receivers was presented by O'Neill and Hudson [6].

Their method for calculating the optical performance is fast and accurate but assumes that the radiation source is a uniform disk. Imhamed, et al. [7] have presented study that aims to develop a 3-D static solar concentrator that can be used as low cost and low energy substitute. Their goals were to design solar concentrator for production of portable hot water in rural India. They used ray tracing software for evaluation of the optical performance of a static 3-D EHC (elliptical hyperboloid concentrator). Optimization of the concentrator profile and geometry 
is carried out to improve the overall performance of system. Kashika and Reddy [8] used satellite dish of $2.405 \mathrm{~m}$ in diameter with aluminium frame as a reflector to reduce the weight of the structure and cost of the solar system. In their solar system, the average temperature of water vapor was $300{ }^{\circ} \mathrm{C}$, when the absorber was placed at the focal point. Cost of their system was US\$ 950. Ouederni, et al. [9] was testing parabolic concentrator of $2.2 \mathrm{~m}$ in diameter with reflecting coefficient 0.85 . Average temperature in their system was $380{ }^{\circ} \mathrm{C}$. Rafeeu and AbKadir [10] have presented simple exercise in designing, building and testing small laboratory scale parabolic concentrators. They made two dishes from acrylonitrile butadiene styrene and one from stainless steel. Three experimental models with various geometrical sizes and diameters were used to analyze the effect of geometry on a solar irradiation. Zhiqiang, et al. [11] presented a procedure to design a facet concentrator for a laboratory-scale research on medium - temperature thermal processes. The facet concentrator approximates a parabolic surface with a number of flat square facets supported by a parabolic frame and having two edges perpendicular to the concentrator axis. A 164-facet concentrator will deliver up to $8.15 \mathrm{~kW}$ of radiative power over $15 \mathrm{~cm}$ radius disk located in the focal plane. Their system had average concentration ratio exceeding 100. Ahmed and Khan [12] presented two prototype parabolic dishes: the Shenandoah dish and the parabolic dish concentrator, in jet propulsion laboratory. The Shenandoah dish was designed to heat silicone oil in one pass to $400{ }^{\circ} \mathrm{C}$. The Shenandoah dish is $7 \mathrm{~m}$ parabolic reflecting dish formed from 21 aluminum petals covered with special reflective layer on one side. The second parabolic dish (JPL parabolic dish concentrator) has parabolic reflector surface with $12 \mathrm{~m}$ diameter. Rebecca, et al. [13] investigated experimental evaluation of ammonia receiver geometries with a $9 \mathrm{~m}^{2}$ dish concentrator. The $20 \mathrm{~m}^{2}$ dish is mirrored with around 2,000 flat mirror tile facets arranged in concentric rings on a parabolic fiber glass support structure. Size of mirror facets is from $5 \mathrm{~cm}$ to $10 \mathrm{~cm}$. Glen, et al. [14] analyzed optical performance of spherical reflecting elements for use with parabolic dish concentrators. This concentrator consists of 54 triangular mirrors. The effective rim angle for the dish is $46^{\circ}$. The 54 units are composed of nine separate panel shapes, each of shapes is duplicated six times. The focal length of system is $13.1 \mathrm{~m}$. They compared the optical performances and manufacturing feasibility of collectors having such a combination of surfaces.

The decision to make solar parabolic concentrator with 12 petals is based on large number of design concepts that are realized in the world. This concept already proved useful in solar techniques, especially in production of heat and electrical energy as well as in trigeneration and polygeneration systems.

The basic idea behind this research is to start with primary concept of solar parabolic concentrator which will generate from $10 \mathrm{~kW}$ to $25 \mathrm{~kW}$ in polygeneration systems. Only with employment of parabolic concentrating systems, it is possible to obtain high temperatures in range from $200{ }^{\circ} \mathrm{C}$ to $800{ }^{\circ} \mathrm{C}$ and high thermal efficiency.

\section{Optical Design of the Solar Parabolic Thermal Concentrator}

The optical design of the solar parabolic thermal concentrator and operation are presented. Optical design is based on parabolic dish with 12 curvilinear trapezoidal petals. Solar dish concentrators are generally concentrators that concentrate solar energy in a small area known as focal point. Dimensions of reflecting surfaces in solar dish concentrator are determined by desired power at maximum levels of insolation and efficiency of collector conversion. Mathematical representation of parabolic concentrator is paraboloid which can be represented as a surface obtained by rotating parabola around axis which is shown in Fig. 1.

Mathematical equations for the parabolic dish solar 


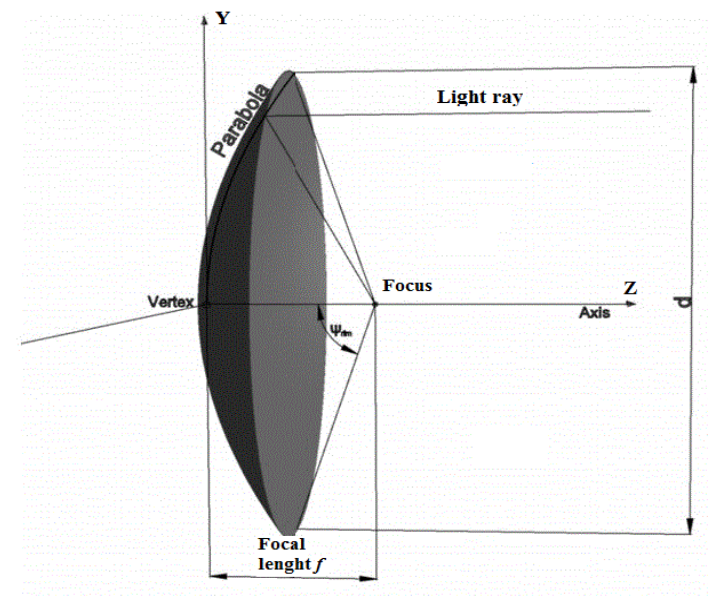

Fig. 1 Ideal shape of parabolic solar concentrator.

concentrator (Fig. 1) in Cartesian and cylindrical coordinate systems are defined as:

$$
\begin{gathered}
\mathrm{x}^{2}+\mathrm{y}^{2}=4 f \mathrm{z} \\
\mathrm{z}=r^{2} / 4 f
\end{gathered}
$$

where, $\mathrm{x}$ and $\mathrm{y}$ are coordinates in aperture plane; and $\mathrm{z}$ is distance from vertex measured along the line parallel with the paraboloid axis of symetry; $f$ is focal length of paraboloid i.e., distance from the vertex to the focus along the aparboloid axis of symmetry. The relationship between the focal length and the diameter of parabolic dish is known as the relative aperture and it defines shape of the paraboloid and position of focal point. The shape of paraboloid can be also defined by rim angle $\psi_{\text {rim }}$. Usually paraboloids that are used in solar collectors have rim angles from $10^{\circ}$ up to $90^{\circ}$. The relationship between the relative aperture and the rim angle is given by:

$$
f / D=\frac{1}{4 \tan \left(\psi_{\text {rim }}{ }^{2}\right)}
$$

The paraboloid with small rim angles have the focal point and receiver at large distance from the surface of concentrator. The paraboloid with rim angle smaller than $50^{\circ}$ is used for cavity receivers while paraboloids with large rim angles are most appropriate for the external volumetric receivers (central receiver solar systems).

The geometric concentration ratio can be defined as the area of the collector aperture $A_{\text {app }}$ divided by the surface area of the receiver $A_{\text {rec }}$ and can be calculated by Eq. (3):

$$
C R_{g}=\left(\sin ^{2} \theta_{a}\right)^{-1}=A_{c} A_{r}^{-1}=A_{a p p} / A_{\text {rec }}
$$

The designed solar parabolic concentrator has geometric concentration ratio $\mathrm{CR}=13,615$.

Flux concentrating ratio can be defined as ratio of flux concentrated in a point $I$ to incident solar flux $I_{b, n}$ :

$$
C R_{\text {flux }}=\frac{I}{I_{b, n}}
$$

\subsection{Design Description of Solar Parabolic Concentrator}

Mechanical design of the solar parabolic concentrator is done in 3D design software CATIA, Dassault systems, USA. Parabolic shape of solar concentrator is obtained by entering $\mathrm{X}$ and $\mathrm{Y}$ coordinates for selected points. For calculation of necessary points that define parabola public domain software Parabola Calculator 2.0 is used. The calculated coordinates (X and $\mathrm{Y}$ ) for designed parabola are shown in Table 1.

Geometrical model of solar parabolic concentrator is parametrically designed from calculated coordinates and it is shown in Fig. 2. Selected model of solar dish concentrator with 12 petals requires very precise definition of parameters during geometrical modelling of system. Results obtained by optical analysis of solar concentration system are very much dependent on the selected method of the geometrical model generation.

A truncated paraboloid of revolution (circular paraboloid) is obtained by rotating the parabola segment about its axis (Fig. 3).

Consider a concentrator consisting of 12 trapezoidal reflective petals of identical non-overlapping trapezoidal segments. 3D model of trapezoidal reflective petal of solar parabolic concentrator is presented in Fig. 4.

Table 1 Coordinates of designed parabola.

\begin{tabular}{llllllll}
\hline$X(\mathrm{~cm})$ & -140.0 & -116.6 & -93.33 & -70.00 & -46.67 & -23.33 & 0.0 \\
$\mathrm{Y}(\mathrm{cm})$ & 35 & 24.31 & 15.56 & 8.75 & 3.89 & 0.97 & 0.0 \\
$\mathrm{X}(\mathrm{cm})$ & 23.33 & 46.67 & 70.00 & 93.33 & 116.67 & 140 & - \\
$\mathrm{Y}(\mathrm{cm})$ & 0.97 & 3.83 & 8.75 & 15.56 & 24.31 & 35.00 & - \\
\hline
\end{tabular}




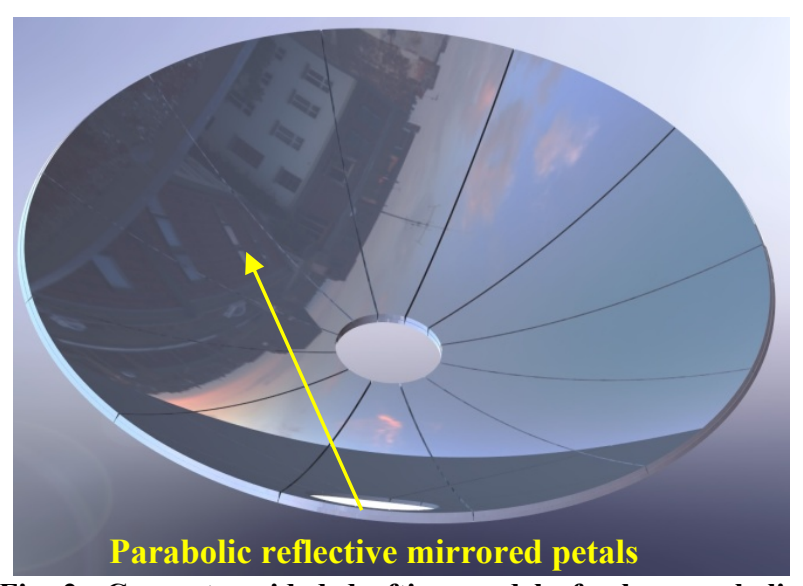

Fig. 2 Computer aided drafting model of solar parabolic dish concentrator.

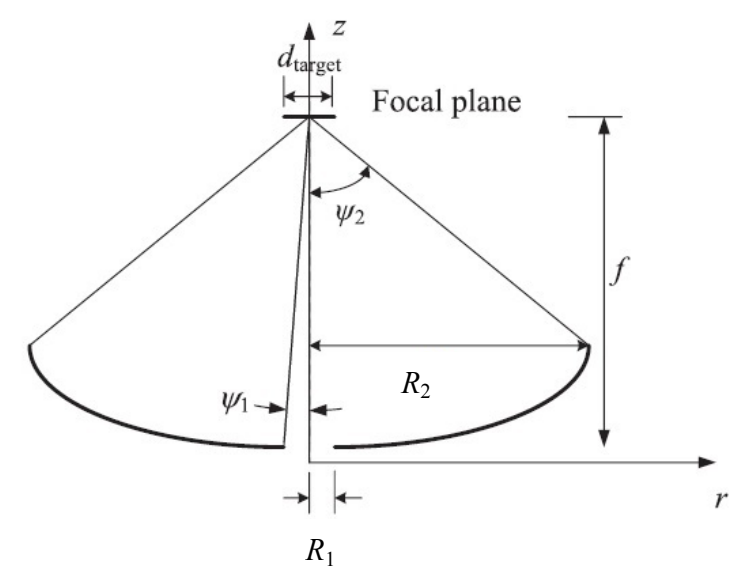

Fig. 3 Schematic of truncated parabola.

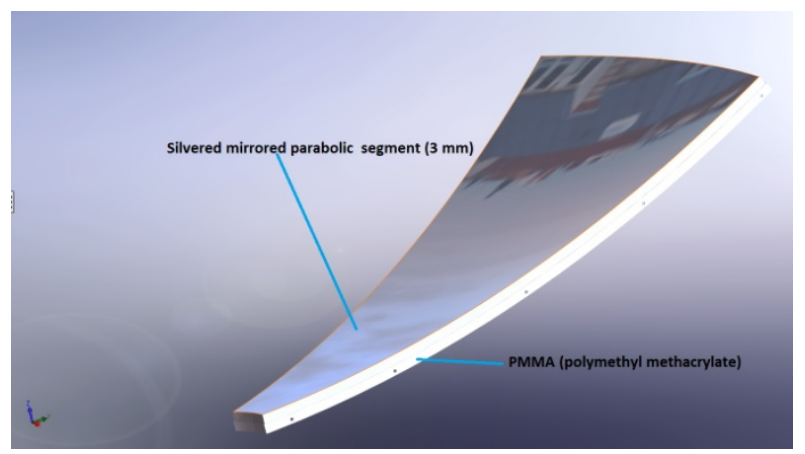

Fig. 4 Trapezoidal reflective petal of solar parabolic dish concentrator.

Detailed design parameters of solar parabolic concentrator are given in Table 2 .

Receiver-absorber is placed in focal area where reflected radiation from solar concentrator is collected. In the process of designing, parabolic solar concentrators one always seek for the minimum size of the receiver. With small receiver size, one can reduce heat losses as well as cost of whole system. Also small receiver size provides increase of absorbed flux on the surface of receiver. This is the way of obtaining greater efficiency in conversion of solar radiation to heat. In our system, receiver-absorber is cylindrical disk with diameter of $24 \mathrm{~mm}$. It is shown in Fig. 5. Within the solar absorber is placed spiral corrugated pipe through which water flows.

Fig. 6 shows the modified solar receiver with solar spiral corrugated pipe absorber in the next design of my investigations. This is the next solar receiver design concentrator.

In this paper, only optical properties of receiver are analysed. In our further research, we plan to model all necessary details of receiver's geometry which are important for conversion of solar energy into heat of fluid that is used for transfer energy.

\section{Optical Analysis of the Solar Parabolic Dish Thermal Concentrator}

For optical analysis of solar parabolic thermal concentrator software TracePro, Lamda Research Corporation, USA is used. First step was importing 3D model designed in CATIA. In TracePro, all material properties are assigned. Twelve trapezoidal reflective petals are defined as standard mirrors with reflective coating. Reflection coefficient was $95 \%$. Receiver was cylinder with diameter $24 \mathrm{~mm}$ placed on 1,400 $\mathrm{mm}$ from vertex of parabolic dish. Absorbing surface was defined as perfect absorber. After definition of geometry of solar, parabolic concentrator radiation source was defined. Radiation source was circular with diameter same as diameter of parabolic dish $(2,800 \mathrm{~mm})$. Radiation source was placed 2,000 mm from vertex of parabolic dish and had circular grid pattern for generating 119,401 rays for Monte Carlo ray tracing. Spatial profile of generated rays was uniform and angular profile was solar radiation. Input parameter for optical analysis is solar irradiance $800 \mathrm{~W} / \mathrm{m}^{2}$. Experiential value for solar irradiation for town of Niš 
Table 2 Design parameters of solar parabolic dish concentrator.

\begin{tabular}{lll}
\hline Parameters & Numerical value & Unit \\
\hline Aperture radius $R_{2}$ & 1.4 & $\mathrm{~m}$ \\
Radius of smaller hole $R_{1}$ & 0.025 & $\mathrm{~m}$ \\
Ideal area of the concentrator $A_{\text {idel }}$ & 6.208 & $\mathrm{~m}^{2}$ \\
The cross section of the opening parabola $A_{\text {proj }}$ & 6.154 & $\mathrm{~m}^{2}$ \\
A sheltered area of the concentrator $A_{\text {shadow }}$ & 0.000452 & $\mathrm{~m}^{2}$ \\
The effective area of the concentrator $A_{\text {ef }}=A_{\text {proj }}-A_{\text {shadow }}$ & 6.1535 & $\mathrm{~m}^{2}$ \\
Receiver diameter & 0.20 & $\mathrm{~m}$ \\
Shape of receiver & Flat circular disc & - \\
Depth of the concentrator & 0.35 & $\mathrm{~m}$ \\
Focal lenght & 1.4 & $\mathrm{~m}$ \\
$\psi_{1}$ & 10 & $\circ$ \\
$\psi_{2}$ & 45 & $\circ$ \\
\hline
\end{tabular}

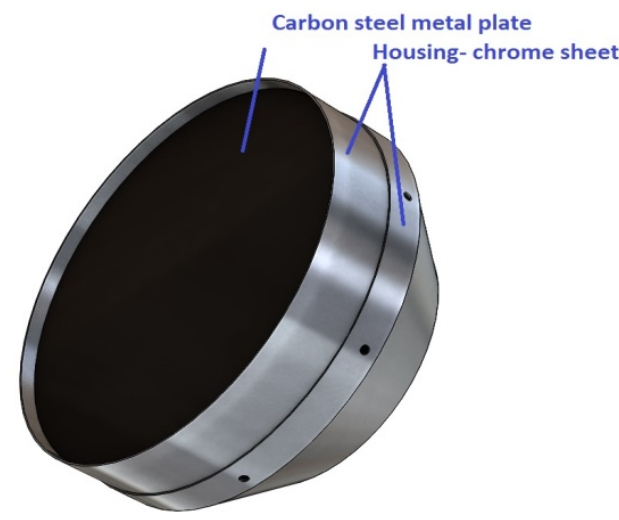

(a)

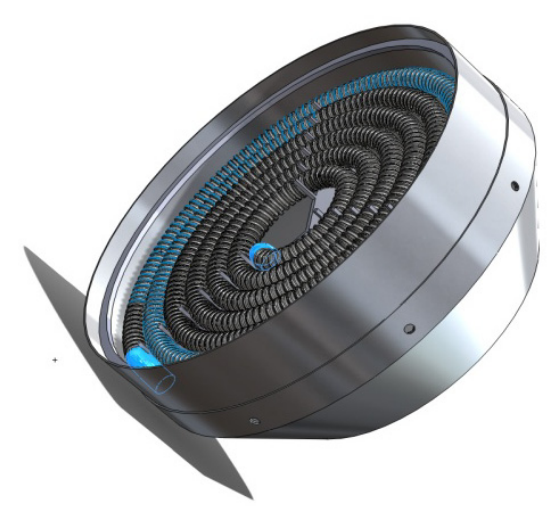

(b)

Fig. 5 Solar thermal receiver - with flat plate circular disc and without (directly irradiated spiral corrugated pipe absorber).

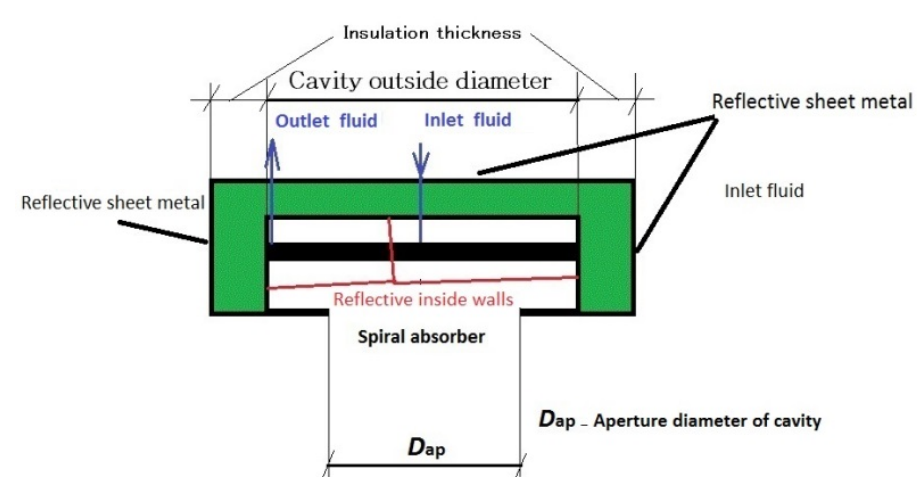

(a)

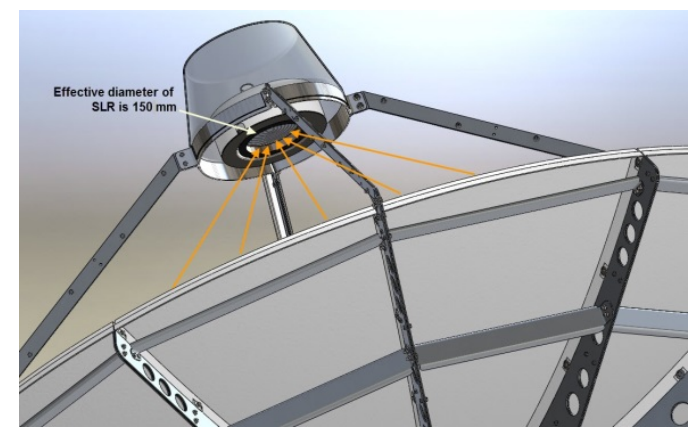

(b)

Fig. 6 (a) Modified solar cavity receiver for solar parabolic dish concentrating collector and (b) concentrated solar radiation at the focus plane.

in Serbia is between $750 \mathrm{~W} / \mathrm{m}^{2}$ and $900 \mathrm{~W} / \mathrm{m}^{2}$. Optical system for solar parabolic concentrator with traced rays is given in Fig. 7. The optical concentration system consists of three objects: solar parabolic dish reflector, radiation source and receptor (solar receiver) in the focus of the parabolic dish concentrator

Optical analysis is done by generating and calculating Monte Carlo ray trace for 119,401 rays. From all emitted rays, only 103,029 rays reached absorber surface which is $82 \%$ rays of emitted rays are 


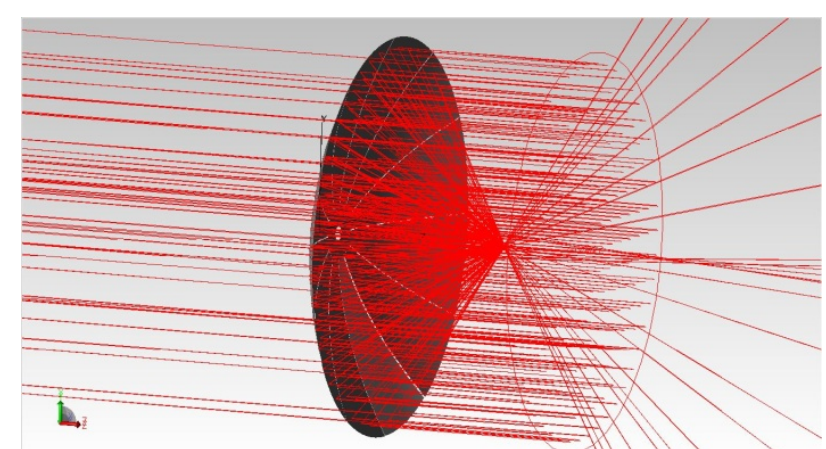

Fig. 7 Optical system of Solar parabolic concentrator with traced rays.

absorbed on receiver. Calculated irradiance for absorbed rays on receiver is from $8.66 \times 10^{-6} \mathrm{~W} / \mathrm{m}^{2}$ to $3.45 \times 10^{7} \mathrm{~W} / \mathrm{m}^{2}$.

Total calculated flux on receiver was $4,031 \mathrm{~W}$. In Fig. 8 is shown total irradiance map for absorbed flux on receiver.

From Fig. 8, one can see that, calculated values for total irradiance are in compliance with theoretically values. In the center of receiver, irradiance is from $3.45 \times 10^{7} \mathrm{~W} / \mathrm{m}^{2}$ to $2 \times 10^{7} \mathrm{~W} / \mathrm{m}^{2}$ and at the periphery of receiver irradiance is from $8 \times 10^{6} \mathrm{~W} / \mathrm{m}^{2}$ to $2 \times 10^{6} \mathrm{~W} / \mathrm{m}^{2}$.

Irradiance diagram at the center of receiver is given in Fig. 9.

From Fig. 9, one can see that, peak irradiance is in the circle with diameter $8 \mathrm{~mm}$ (from $-4 \mathrm{~mm}$ to $4 \mathrm{~mm}$ ). Very good irradiance is in the circle with diameter $16 \mathrm{~mm}$. Irradiance diagram at the periphery of receiver is given in Fig. 10.

From Fig. 10, one can see that, irradiance diagram at periphery of receiver is still rather good with peak values of irradiance in the circle diameter $4 \mathrm{~mm}$. Very good values of irradiance is in the circle with diameter $16 \mathrm{~mm}$. From Figs. 9 and 10, one can see that, irradiance has very good values in the circle with diameter $16 \mathrm{~mm}$ for center and periphery and that only peak values change from center to periphery.

\section{Conclusions}

This paper presents optical analysis of the solar parabolic concentrator using the ray-tracing software

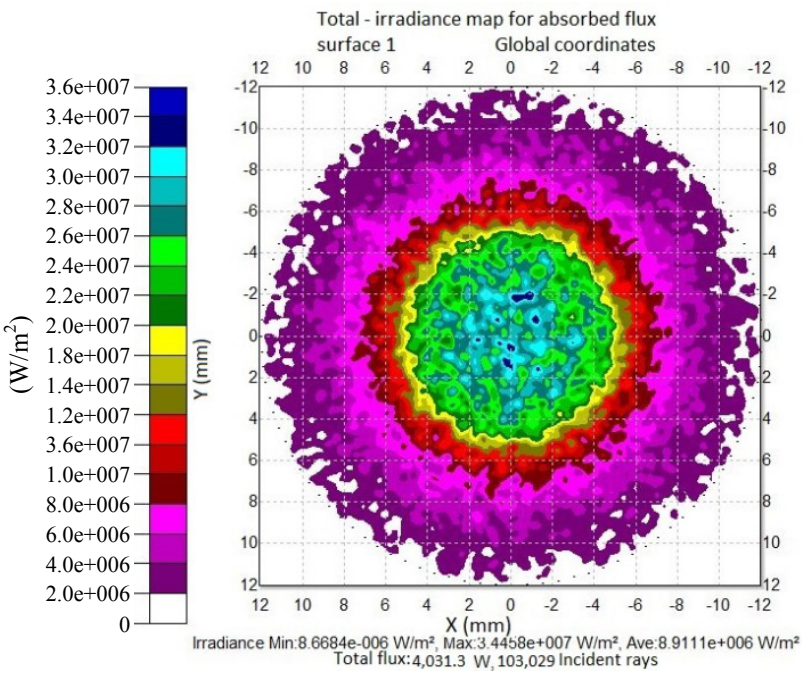

Fig. 8 Irradiance map for absorbed flux on receiver.

Irradiance diagram at the center of receiver $y=0 \mathrm{~mm}$

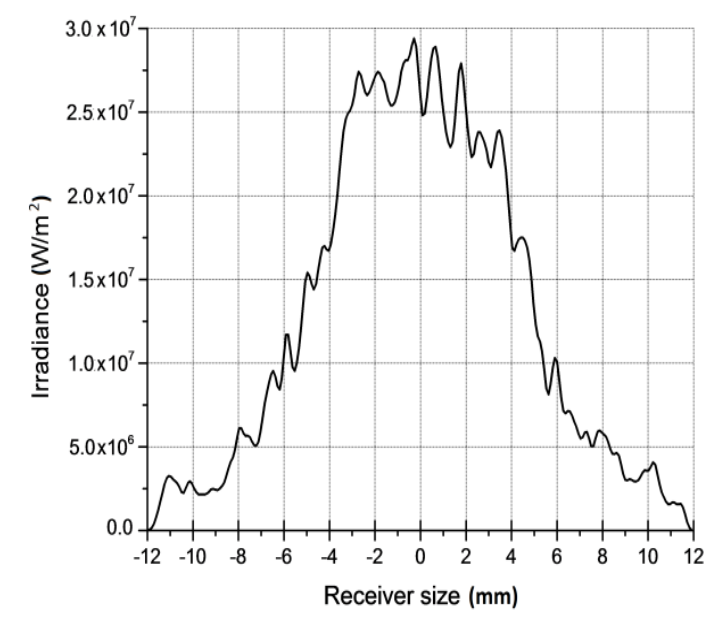

Fig. 9 Irradiance diagram at the center of receiver.

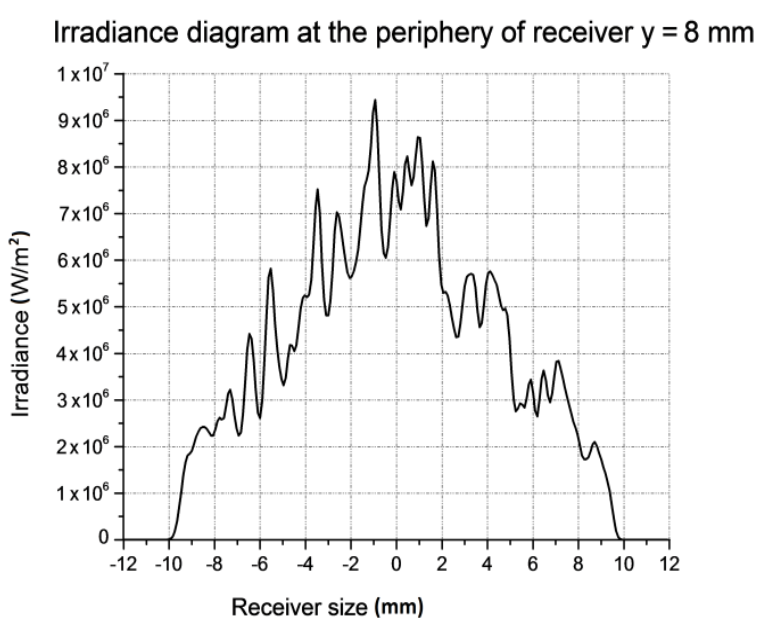

Fig. 10 Irradiance diagram at the periphery of receiver. 
TracePro. One can see that, results obtained from optical design of solar parabolic concentrator are satisfactory. Total flux in focal area is good. Irradiance distribution for absorbed flux is relatively uniform for small area for absorber. As a next step, various analysis and simulations of the model are planed. Among others are variation of number of petals, size of petals and shape of petals. In future development of optimization, method is planned. This optimization method will make it possible to find optimal geometrical and optical parameters of the various types of solar parabolic dish concentrators as well as geometrical, optical and thermal parameters of receivers-absorbers.

\section{Acknowledgments}

This paper is done within the research framework of research projects: III42006-research and development of energy and environmentally highly effective polygeneration systems based on renewable energy resources and III45016-fabrication and characterization of nano-photonic functional structrues in biomedicine and informatics. Both projects are financed by Ministry of Education, Science and Technological Development of Republic of Serbia. Authors acknowledge to Lambda Research Corporation for allowing to use software TracePro for $\mathrm{PhD}$ thesis research of Saša Pavlović.

\section{References}

[1] Badran, A. A., and Yousef, I. A. 2010. "Portable Solar Cooker and Water Heater." Energy Conversion and Management 51 (8): 1605-9.

[2] Joshi, A. S., Dincer, I., and Reddy, B. V. 2011. "Solar Hydrogen Production: A Comparative Performance Assessment." International Journal of Hydrogen Energy 36 (17): 11246-57.
[3] Furler, P., Scheffe, J. R., and Steinfeld, A. 2012. "Syngas Production by Simultaneous Splitting of $\mathrm{H}_{2} \mathrm{O}$ and $\mathrm{CO}_{2}$ via Ceria Redox Reactions in a High-Temperature Solar Reactor." Energy \& Environmental Science 5 (3): 6098-103.

[4] Mancini, T., Heller, P., Butler, B., Osborn, B., Schiel, W., Goldberg, V., Buck, R., Diver, R., Andraka, C., and Moreno, J. 2003. "Dish-Stirling Systems: An over View of Development and Status." Journal of Solar Energy Engineering-Transactions of the ASME 125 (2): 135-51.

[5] Mills, D. 2004. "Advances in Solar Thermal Electricity Technology.” Solar Energy 76 (1-3): 19-31.

[6] O’Neill, M. J., and Hudson, S. L. 1978. "Optical Analysis of Paraboloidal Solar Concentrators." In Proceedings of the 1978 Annual Meeting, 855.

[7] Imhamed, M. S. A., Tadhg, S. O., Reddy, K. S., and Tapas, K. M. 2013. "An Optical Analysis of a Static 3-D Solar Concentrator." Solar Energy 88 (February): 57-70.

[8] Kaushika, N. D., and Reddy, K. S. 2000. "Performance of Low Cost Solar Paraboloidal Dish Steam Generating." Energy Conversion \& Management 41 (7): 713-26.

[9] Ouederni, A. R. E., Ben Salah, M., Askri, F., and Aloui, F. 2009. "Experimental Study of a Parabolic Solar Concentrator." Revue des Renouvelables 12 (3): 395-404.

[10] Rafeeu, Y., and AbKadir, M. Z. A. 2012. "Thermal Performance of Parabolic Concentrators under Malysian Environment: A Case Study." Renewable and Sustainable Energy Reviews 16 (6): 3826-35.

[11] Zhiqiang, L., Justin, L., and Wojciech. L. 2012. "Optical Design of a Flat Solar Concentrator." Solar Energy 86 (6): 1962-6.

[12] Hasnat, A., Ahmed, P., and Khan, K. A. 2011. "Numerical Analysis for the Thermal Design of a Paraboloidal Solar Concentratong Collector." International Journal of Natural Sciences 1 (3): 68-74.

[13] Rebecca, D., Keith, L., Greg, B., and John, P. 2012. “An Experimental Study of Ammonia Receiver Geometries for Dish Concentrators." Journal of Solar Energy Engineering 134 (4): 945-55.

[14] Glen, J., Keith, L., and Andreas, L. 2003. "Optical Performance of Spherical Reflecting Elements for Use with Paraboloidal Dish Concentrators." Solar Energy 74 (2): 133-40. 